\title{
What did ASCA see in the GRB 970828 afterglow?
}

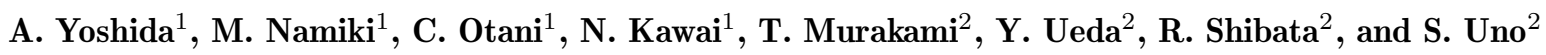 \\ 1 The Institute of Physical and Chemical Research, 2-1, Hirosawa, Wako, Saitama 351-0198, Japan \\ e-mail: ayoshida@postman.riken.go.jp \\ 2 Institute of Space and Astronautical Science, 3-1-1, Yoshinodai, Sagamihara, Kanagawa 229-8510, Japan
}

Received January 21; accepted July 5, 1999

\begin{abstract}
The ASCA satellite made a quick followup observation of GRB 970828 and detected its fading X-ray afterglow at the position localized by the All-Sky Monitor on the Rossi XTE satellite. Because of the quickness, the afterglow was still rather bright $\left(\sim 410^{-13} \mathrm{ergs} \mathrm{cm}^{-2} \mathrm{~s}^{-1}\right.$ : $2-10 \mathrm{keV}$ ), and ASCA obtained the data of good quality. We found the spectral features of the absorption which implies a column density of $4.110^{21} \mathrm{~cm}^{-2}$ at $z=0$, together with an emission line at $\sim 5 \mathrm{keV}$. The line could be red shifted $\mathrm{Fe} \mathrm{K}_{\alpha}$ line. Hence, it suggests a relatively small red shift $z \approx 0.33$ for this GRB.
\end{abstract}

Key words: gamma-rays: bursts

\section{Introduction}

Among nine GRB afterglows which displayed optical counterparts, the red-shift of the source is found from the optical spectroscopy for three GRBs, 970508, 971214 and 980703 (Metzger et al. 1997; Kulkarni et al. 1998; Djorgovski et al. 1998), and one, GRB 980329, was implied to be $z \sim 5$ by Fruchter (1998) from its fluxes in $K-R$ band. Another noteworthy GRB is GRB 980425 which is suggested to be associated with a SN Ic, SN1998bw, at $z=0.0085$ (Galama et al. 1998).

In contrast to these events, the burst of 28 August 1997 (GRB 970828) displayed an afterglow only in an $\mathrm{X}$-ray band and accompanied no optical transient down to $R=23.8$ (Groot et al. 1998). This GRB was detected by ASM/RXTE on August 28.73931, 1997 UT and localized into a small region with $2^{\prime} \times 5^{\prime}$ accuracy (Remillard et al. 1997; Smith et al. 1997). A subsequent scan by the PCA/RXTE (Proportional Counter Array) found an X-ray afterglow of $0.5 \mathrm{mCrab}(2-10 \mathrm{keV})$ about four hours after the burst detection (Marshall et al. 1997). Here we report the detection of an afterglow of GRB 970828, and its X-ray properties obtained with ASCA.

Send offprint requests to: A. Yoshida

\section{Observation and analysis}

An ASCA observation of GRB 970828 in the $0.5-10 \mathrm{keV}$ range as a Target of Opportunity Observation (TOO) was conducted soon after the alerts from the RXTE team based on the ASM/RXTE detection. The observation with net exposure time of $\sim 36 \mathrm{ksec}$ was performed during a period of August 29.91 - August 30.85 UT beginning at 1.17 days after the GRB. Both two scientific instruments on ASCA, the SIS and the GIS detector, saw an X-ray source of an average $2-10 \mathrm{keV}$ flux of $F_{\mathrm{X}} \sim$ $410^{-13} \mathrm{erg} \mathrm{s}^{-1} \mathrm{~cm}^{-2}$ within the combined source error region given by ASM/RXTE and IPN (Smith et al. 1997; Hurley et al. 1997; Murakami et al. 1997). The position of the X-ray source was determined at R.A. $=18^{\mathrm{h}} 08^{\mathrm{m}} 32^{\mathrm{s}} .2$ and Decl. $=+59^{\circ} 18^{\prime} 54^{\prime \prime}(\mathrm{J} 2000)$ with a $90 \%$ error radius of 0.5 by an image analysis on the datasets.

\subsection{X-ray flux variability}

The X-ray source clearly displayed a fading behavior during the observation. The flux in $2-10 \mathrm{keV}$ range estimated assuming a power-law spectrum with the photon index of -2 decayed from $\sim 110^{-11} \mathrm{ergs}^{-2} \mathrm{~s}^{-1}$ (the PCA observation at $\left.t \sim 1.410^{4} \mathrm{~s}\right)$ to $\sim 310^{-13} \mathrm{ergs} \mathrm{cm}^{-2} \mathrm{~s}^{-1}$ (at the end of the ASCA observation at $t \sim 1.710^{5} \mathrm{~s}$ ) where $t$ is an elapsed time since the burst. However,the ASCA data indicate a variability of the source rather than a simple power-law like decay. Figure 1 shows the SIS count rate together with the "best-fit" power-law fading model: $t^{-1.44 \pm 0.07}$ (solid line) based on the PCA/RXTE (Marshall et al. 1997) and the ASCA observation. A peak structure appears around $t=1.2510^{5} \mathrm{~s}$.

\subsection{Afterglow spectra}

Spectra of the source were made for three periods during the observation indicated by dashed lines labeled "A", "B" and "C" in Fig. 1. We made fittings with a model "absorbed power-law" $\left(\exp \left(-N_{\mathrm{H}} \sigma(E)\right) * E^{-\alpha}\right)$, and found a large absorption column density, $N_{\mathrm{H}}$ in the best fit 


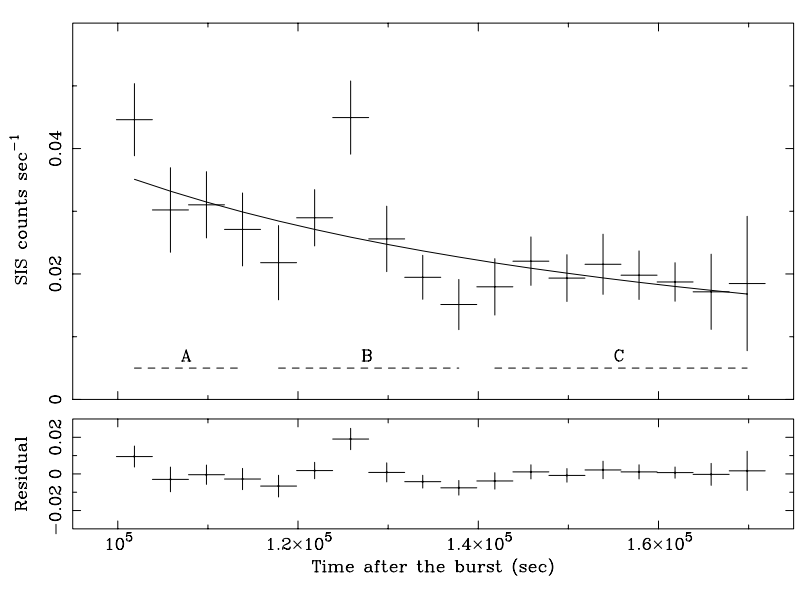

Fig. 1. SIS light curve with time bins of 4000 s. It displays the time variation of the source brightness. A simple power-law decay model cannot fit the data; the solid line shows the "bestfit" model. Dashed lines labeled "A", "B" and "C" indicate the intervals for which spectral studies are done

for the period "C". This is $4.1_{-1.6}^{+2.1} 10^{21} \mathrm{~cm}^{-2}$ which is larger than the galactic value towards the source direction, $3.410^{20} \mathrm{~cm}^{-2}$, estimated by COLDEN at The Einstein On-Line Service, Smithsonian Astrophysical Observatory. For other periods, however, derived column densities are consistent with the galactic values.

Another remarkable result is an excess feature around $5 \mathrm{keV}$ in the spectrum of "B" which can be represented by a gaussian line (see Fig. 2). Introducing a line improves the fitting by $\Delta \chi^{2}=11.15$ with three additional line parameters, and results in $\chi^{2}$ of 58.85 for 58 dof. An F-test gives the confidence of $98.3 \%$. The best fit line centroid is $E_{\text {line }}=5.04_{-0.31}^{+0.23} \mathrm{keV}$, the line width $\sigma=0.31_{-0.31}^{+0.38} \mathrm{keV}$, and the line flux $F_{\mathrm{Fe}}=(1.5 \pm 0.8) 10^{-13} \mathrm{ergs} \mathrm{cm}^{-2} \mathrm{~s}^{-1}$. While, for the spectra of "A" and "C", the model including a line results in its normalization consistent with zero. The upper-bounds (90\% confidence) of line flux are $9.510^{-14} \mathrm{ergs} \mathrm{cm}^{-2} \mathrm{~s}^{-1}$ and $5.610^{-14} \mathrm{ergs} \mathrm{cm}^{-2} \mathrm{~s}^{-1}$ for "A" and "C"respectively. This may imply that the line intensity changed during the observation.

\section{Summary}

ASCA saw a fading X-ray afterglow from GRB 970828. However its flux not monotonically decayed but displayed a "flare activity" around $t=1.2510^{5} \mathrm{~s}$. The BeppoSAX observed an X-ray flux variability also in the GRB afterglow of GRB 970508 (Piro et al. 1997). It is a rather broad $\left(\delta t>10^{5} \mathrm{~s}\right.$ at $\left.t \approx 10^{5} \mathrm{~s}\right)$ "bursting activity", while the activity seen with ASCA is narrower in time; $\delta t / t \sim 0.05$. Fenimore et al. (1998) suggests that this small $\delta t / t$ implies a small surface filling factor similar to the GRB activities themselves and there would be fine structures in the relativistic shell much smaller than $\Gamma^{-1}$ where $\Gamma$ is the bulk Lorentz factor.

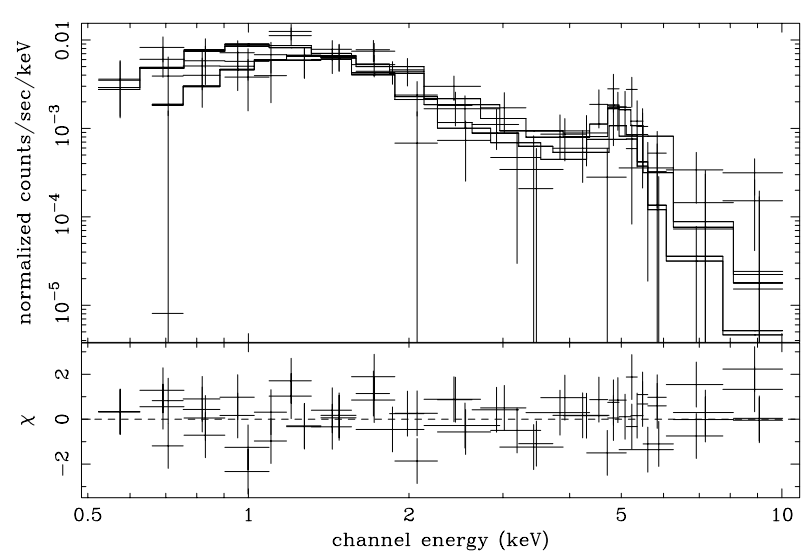

Fig. 2. The X-ray spectrum for the period (B) shown in Fig. 1 fitted with the model of "absorbed power-law" $\left(\exp \left(-N_{\mathrm{H}} \sigma(E)\right) * E^{-\alpha}\right)$

ASCA discovered in the afterglow spectra the evidence of a large absorption column density and an emission line possibly caused by the $\mathrm{Fe} \mathrm{K}_{\alpha}$ line emissions red shifted by $z=0.33$. This would be the first GRB with its red shift determined only by the X-ray observation if this interpretation is true. A detection of a red-shifted $\mathrm{Fe}$ emission line is also reported by the BeppoSAX team for the GRB 970508 afterglow (Piro et al. 1999a; Piro et al. 1999b). The Fe line seen in the GRB 970828 was variable in time with the time scale of $\sim 10000 \mathrm{~s}$ and appeared $\sim 10^{5} \mathrm{~s}$ after the burst. This suggests a rather small line forming region of $\sim 10^{14}-10^{15} \mathrm{~cm}$ and implies a dense matter $\left(n>10^{12}\right.$ for the matter with the solar abundance) in the vicinity of the source if the line is formed by the photoionization-recombination process or the "reflection" mechanism (Yoshida et al. 1999).

Acknowledgements. We thank the ASCA team for allowing this TOO.

\section{References}

Djorgovski S.G., et al., 1998, ApJ 508, L17

Fenimore E.E., et al., 1999, ApJ 512, 683

Fruchter A.S., 1998, astro-ph/9810224

Galama T.J., et al., 1998, Nat 395, 670

Groot P.J., et al., 1998, ApJ 493, L27

Hurley K., et al., 1997, IAU Circ., No. 6728

Kulkarni S.R., et al., 1998, Nat 393, 35

Marshall F.E., et al., 1997, IAU Circ., No. 6727

Metzger M.R., et al., 1997, Nat 387, 878

Murakami T., et al., IAU Circ., No. 6732 (1997)

Piro L., et al., 1997, A\&A 331, L41

Piro L., et al., 1999a, ApJ 514, L73

Piro L., et al., 1999b, A\&A (in this volume)

Remillard R., et al., 1997, IAU Circ., No. 6726

Smith D., et al., 1997, IAU Circ., No. 6728

Yoshida A., et al., 1998, ApJ (submitted) 